\title{
Stroboscopic quantum optomechanics
}

\author{
Matteo Brunelli, ${ }^{1}$ Daniel Malz $\odot,{ }^{2}$ Albert Schliesser $\odot,{ }^{3,4}$ and Andreas Nunnenkamp ${ }^{1}$ \\ ${ }^{1}$ Cavendish Laboratory, University of Cambridge, Cambridge CB3 OHE, United Kingdom \\ ${ }^{2}$ Max-Planck-Institut für Quantenoptik, Hans-Kopfermann-Strasse 1, D-85748 Garching, Germany \\ ${ }^{3}$ Niels Bohr Institute, University of Copenhagen, 2100 Copenhagen, Denmark \\ ${ }^{4}$ Center for Hybrid Quantum Networks (Hy-Q), Niels Bohr Institute, University of Copenhagen, 2100 Copenhagen, Denmark
}

(Received 19 March 2020; accepted 13 May 2020; published 28 May 2020)

\begin{abstract}
We consider an optomechanical cavity that is driven stroboscopically by a train of short pulses. By suitably choosing the interpulse spacing we show that ground-state cooling and mechanical squeezing can be achieved, even in the presence of mechanical dissipation and for moderate radiation-pressure interaction. We provide a full quantum-mechanical treatment of stroboscopic backaction-evading measurements, for which we give a simple analytic insight, and discuss preparation and verification of squeezed mechanical states. We further consider stroboscopic driving of a pair of noninteracting mechanical resonators coupled to a common cavity field, and show that they can be simultaneously cooled and entangled. Stroboscopic quantum optomechanics extends measurement-based quantum control of mechanical systems beyond the good-cavity limit.
\end{abstract}

DOI: 10.1103/PhysRevResearch.2.023241

\section{INTRODUCTION}

Cavity optomechanics has proven extremely successful in controlling nanoscale and microscale mechanical motion at the quantum level [1]. Among the key achievements is the demonstration of ground-state cooling [2,3], mechanical squeezing [4-6], and mechanical entanglement [7]. Most of these milestones have been obtained in sideband-resolved optomechanical systems operating in the continuous-wave or amplitude-modulated (two-tone) regime, where a notion of stationary regime can be defined, in some suitable rotating frame. Going beyond steady-state operation may be beneficial for several reasons, e.g., it allows to circumvent stability requirements. Sideband-resolved optomechanical systems driven by long pulses have been considered both for controlling mechanical motion [8-11] and as a model of quantum interface between flying quantum carriers; for instance, entanglement between microwave and mechanical degrees of freedom [12], and quantum state transfer [13] have been demonstrated in this regime.

Pulsed protocols can also lift the stringent requirement of sideband resolution. By employing pulses much shorter than the mechanical period, quantum state preparation and readout, e.g., of low-entropy and squeezed mechanical states [14-20], as well as optomechanical and all-mechanical entanglement [21] can in principle be achieved. However, in order to neglect nonunitary processes, coherent operations are restricted to very short times (also less than a single mechanical cycle). The conditional preparation of quantum states with few pulses

Published by the American Physical Society under the terms of the Creative Commons Attribution 4.0 International license. Further distribution of this work must maintain attribution to the author(s) and the published article's title, journal citation, and DOI. also requires large interaction strengths, which has so far prevented pulsed optomechanics to attain the quantum regime [22]. Only very recently, pulsed operation close to the quantum regime has been demonstrated in a setup based on a photonic crystal nanobeam [23].

In this work we take a different approach and study the conditional dynamics of an optomechanical system driven by a train of pulses. We show that this new regime-stroboscopic quantum optomechanics - is effective to prepare and verify quantum states of mechanical motion beyond the sidebandresolved regime. In particular, by suitably choosing the spacing between the pulses, ground-state cooling and squeezing of a single mechanical resonator can be achieved, as well as collective cooling and entanglement of two nondegenerate resonators (radiation-pressure coupled to a common cavity mode).

Compared with single-pulse protocols, our approach has the distinct advantage to allow for a cumulative effect of the measurements over many mechanical cycles, thus considerably relaxing the requirement on the optomechanical coupling strength. This however requires including mechanical dissipation in the description of the dynamics, as opposed to Refs. [15-19]. Due to the competition between radiationpressure interaction and mechanical dissipation, the mechanical system eventually settles into a steady state, albeit a periodic one. For such a stroboscopic steady state, we provide simple analytic expressions for the conditional state. From this point of view, our work draws an interesting connection between the conditional dynamics of periodically measured systems and the Floquet theory of optomechanics [24,25].

Our study is inspired by early works in backaction-evading (BAE) measurements, where the stroboscopic dynamics of mechanical transducers was studied for the detection of weak classical signals [26-30]. We provide a full quantummechanical treatment of stroboscopic BAE measurements [31,32], which was so far missing. We discuss in detail 
corrections to the ideal measurement regime stemming from thermal decoherence and the finite length of each pulse. Notably, we show that including the latter effect, usually considered detrimental, enables preparing pure mechanical squeezed states and optomechanical entanglement. In short, we show that stroboscopic quantum optomechanics bypasses the need for strong measurements and provides an effective and versatile tool for measurement-based quantum control of mechanical states.

The rest of the paper is organized as follows: in Sec. II we describe the system and derive an effective model of the dynamics based on stroboscopic measurements. The predictions of this model for stroboscopic squeezing and cooling of mechanical motion are presented in Secs. III and IV, respectively. In Sec. V we discuss engineering squeezed quantum states in connection with stroboscopic BAE measurements of mechanical motion. In Sec. VI we implement verification of the conditional state via retrodiction. In Sec. VII we extend stroboscopic quantum optomechanics to noninteracting mechanical resonators coupled to a common cavity field and show that they can be simultaneously cooled and entangled. In Sec. VIII we discuss some experimentally relevant considerations for implementing our ideas. Finally, Sec. IX collects conclusive remarks and provides an outlook.

\section{A SIMPLE MODEL OF STROBOSCOPIC CONDITIONAL DYNAMICS}

We consider a standard optomechanical system where the position $\hat{x}$ of a mechanical oscillator of frequency $\omega_{m}$ modulates the frequency of a cavity mode $\hat{a}$ of linewidth $\kappa$ [1]. The cavity is illuminated with a train of short coherent pulses of length $\tau$ much smaller than the mechanical period, i.e., $\omega_{m} \tau \ll 1$. The number of photons $N_{\mathrm{p}}$ in each pulse is large enough to make linearization of the optomechanical interaction an excellent approximation, such that we have $(\hbar=1)$

$$
\hat{H}_{I}(t)=-g(t) \hat{X}_{c}\left(\hat{X}_{m} \cos \omega_{m} t+\hat{P}_{m} \sin \omega_{m} t\right),
$$

where $g$ is the pulsed coupling constant, $\hat{X}_{c}=\left(\hat{a}+\hat{a}^{\dagger}\right) / \sqrt{2}$, and we expressed the mechanical position in terms of the slowly varying quadratures $\hat{X}_{m}, \hat{P}_{m}$. During the interaction time the coupling induces the unitary evolution

$$
\hat{U}\left(t, t_{0}\right)=\mathcal{T} \exp \left\{-i \int_{t_{0}}^{t} d t_{1} \hat{H}_{I}\left(t_{1}\right)\right\} .
$$

For a very short pulse the harmonic motion can be neglected and the unitary evolution can be approximated as

$$
\hat{U} \approx e^{i \chi \hat{X}_{m} \hat{X}_{c}},
$$

where $\chi$ quantifies the strength of the interaction. An estimate of the latter for a fast cavity in the adiabatic limit $\kappa \gg$ $\tau^{-1}$ yields $\chi=2 g_{0} \sqrt{N_{\mathrm{p}} \tau / \kappa}$, where $g_{0}$ is the single-photon optomechanical coupling. As we show in Appendix A, in this limit the interaction is defacto instantaneous, in that mixing between the two mechanical quadratures is fully neglected. Equation (3) realizes a quantum nondemolition (QND) gate between the optical and mechanical amplitudes [33]: $\hat{X}_{c}, \hat{X}_{m}$ are left untouched and information about them is acquired by the conjugate quadratures $\hat{U}^{\dagger} \hat{P}_{m(c)} \hat{U}=\hat{P}_{m(c)}+\chi \hat{X}_{c(m)}$. The probability of recording a value $P_{c}$ of the optical phase quadrature after such interaction is given by

$$
\begin{aligned}
\operatorname{Pr}\left(P_{c}\right) & =\operatorname{Tr}\left[\left|P_{c}\right\rangle\left\langle P_{c}\left|\otimes \mathbb{1}_{m} \hat{U}\right| 0\right\rangle\langle 0| \otimes \hat{\varrho}_{m} \hat{U}^{\dagger}\right] \\
& =\operatorname{Tr}_{m}\left[\hat{\Upsilon}^{\dagger} \hat{\Upsilon} \hat{\varrho}_{m}\right],
\end{aligned}
$$

where the cavity starts off in the vacuum and the resonator in an arbitrary state $\hat{\varrho}_{m}$. In the second line of Eq. (4) we have rewritten the probability by introducing the family of Kraus operators $\hat{\Upsilon}\left(P_{c}\right)=\left\langle P_{c}|\hat{U}| 0\right\rangle$, elements of the positive operator-valued measure (POVM) $\left\{\hat{\Upsilon}^{\dagger} \hat{\Upsilon}\right\}_{P_{c}}$, that satisfy $\hat{\Upsilon}^{\dagger} \hat{\Upsilon} \geqslant 0, \int d P_{c} \hat{\Upsilon}^{\dagger} \hat{\Upsilon}=\mathbb{1}_{m}$. An explicit expression for $\hat{\Upsilon}$ is given by

$$
\hat{\Upsilon}\left(P_{c}\right)=\frac{1}{\pi^{1 / 4}} e^{-\frac{1}{2}\left(P_{c}-\chi \hat{X}_{m}\right)^{2}},
$$

which shows that the effect of the pulse on the mechanics is akin to a generalized position measurement. This expression has been first used to model momentum diffusion in continuous weak measurements [34]. Later, it was employed to model an optomechanical system driven by a single strong pulse, i.e., the regime of pulsed quantum optomechanics [14]. From Eq. (5) we notice that, when acting on a pure state, the measurement operator multiplies the wave function by a Gaussian function of width $\chi^{-2}$ and centered around the position $P_{c} / \chi$; by increasing the interaction strength $\chi$, the wave function thus gets increasingly localized in position. Upon recording the outcome $P_{c}$, the mechanical density matrix is transformed as

$$
\hat{\varrho}_{m} \rightarrow \frac{1}{\operatorname{Pr}\left(P_{c}\right)} \hat{\Upsilon}\left(P_{c}\right) \hat{\varrho}_{m} \hat{\Upsilon}\left(P_{c}\right)^{\dagger},
$$

which is the conditional, or postmeasurement state.

When the pulse is off, environment-induced decoherence is affecting the otherwise free evolution of the mechanical resonator. The dynamics is governed by

$$
\mathcal{L} \hat{\varrho}_{m}=-i\left[\hat{H}_{0}, \hat{\varrho}_{m}\right]+\gamma(\bar{n}+1) \mathcal{D}[\hat{b}] \hat{\varrho}_{m}+\gamma \bar{n} \mathcal{D}\left[\hat{b}^{\dagger}\right] \hat{\varrho}_{m},
$$

where $\bar{n}$ and $\gamma$ are the mean occupation and damping rate of the mechanical bath and $\hat{b}$ is the annihilation operator associated with mechanical quadratures. The evolution over a finite amount of time is given by the map $\hat{\Phi}_{\text {th }}=e^{\mathcal{L} t}$. The pulsed interaction (6) and the free-evolution-plus-dissipation (7) form the "unit cell" of our stroboscopic model, which can be thought of as a repetition of these two elementary steps; see Fig. 1(c). As we discuss below, when concatenating many such steps one is free to choose the spacing between two subsequent pulses. Over this time (i) the mechanical mode picks up a phase, which determines which quadrature is measured at the next interaction, and (ii) the mechanics exchanges phonons with the thermal environment. In particular, the presence of the latter contribution-neglected in previous studies [15-19] — competes with the measurement, eventually leading to a nonequilibrium steady state.

\section{From measurement-induced evolution to deterministic completely positive maps}

A great simplification comes from assuming that both the measurement and the dissipation act on a Gaussian state, in which case their output is a Gaussian state, too $[33,35,36]$. 
(a)

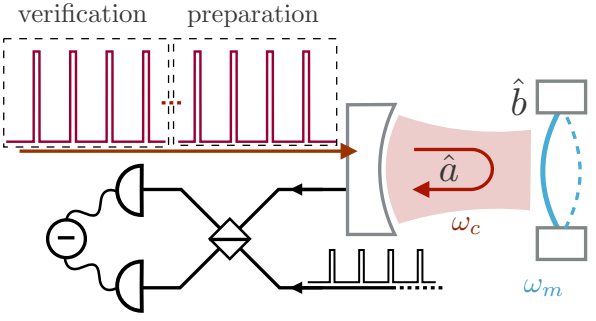

(c)

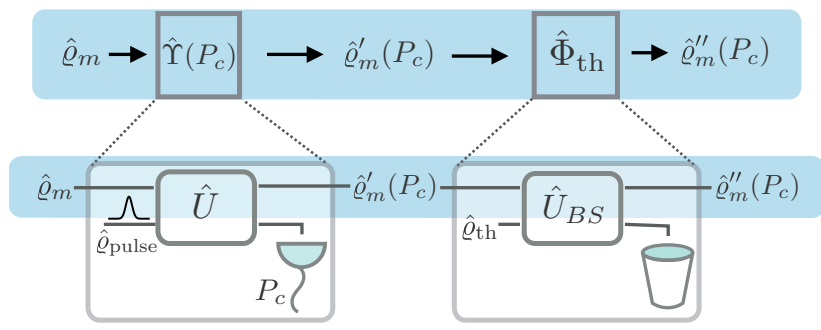

(d)

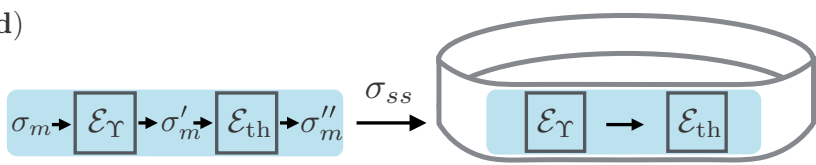

FIG. 1. (a) An optomechanical cavity $(\hat{a})$ is driven by a train of short pulses. After having interacted with the mechanical resonator $(\hat{b})$, the pulses are measured in reflection. Suitably choosing the spacing between the pulses, the setup implements either a stroboscopic backaction-evading (BAE) measurement or measurement cooling. Mechanical squeezing and ground-state cooling can be respectively achieved, conditional on the measurement record. A second train of pulses is used to verify the conditional state. (b) Considering instead two mechanical modes $\hat{b}_{1}, \hat{b}_{2}$, mechanical entanglement and collective cooling can be obtained. (c) Elementary sequence of the stroboscopic protocol: an optical pulse, modeled by a generalized position measurement, is followed by free evolution and mechanical dissipation (see Sec. II for details). (d) For Gaussian input states both measurement and dissipation induce a deterministic transformation of the mechanical covariance matrix. Repeating this sequence yields a stroboscopic steady state, which is invariant under the joint action of measurement and dissipation.

For the case of a Gaussian measurement, such as the quadrature measurement in Eq. (4), the postmeasurement state (6) depends on the measurement outcome only through the first moments or, equivalently, the measurement-induced evolution of the second moments is deterministic; this is a general feature of Gaussian measurements [37]. Therefore, the effect of the measurement can be cast in the form of a deterministic map $\mathcal{E}_{\Upsilon}$ for the second statistical moments [38]. The action of this map $\sigma^{\prime}=\mathcal{E}_{\Upsilon}(\sigma)$ on the mechanical covariance matrix $\sigma$ (with variance $\sigma_{X_{m}}, \sigma_{P_{m}}$ and covariance $\sigma_{X_{m} P_{m}}$ ) is given by

$$
\begin{aligned}
\sigma_{X_{m}}^{\prime} & =\frac{\sigma_{X_{m}}}{1+2 \chi^{2} \sigma_{X_{m}}}, \\
\sigma_{P_{m}}^{\prime} & =\frac{\chi^{2}}{2}+\frac{\sigma_{P_{m}}+2 \chi^{2}\left(\sigma_{X_{m}} \sigma_{P_{m}}-\sigma_{X_{m} P_{m}}^{2}\right)}{1+2 \chi^{2} \sigma_{X_{m}}}, \\
\sigma_{X_{m} P_{m}}^{\prime} & =\frac{\sigma_{X_{m} P_{m}}}{1+2 \chi^{2} \sigma_{X_{m}}} .
\end{aligned}
$$

We explicitly see that the stochastic component of the measurement $\left(P_{c}\right)$ is absent from the above expressions. The first and second expression describe the reduction of the variance along $\hat{X}_{m}$, and the increased fluctuations of the conjugate quadrature due to the quantum backaction, respectively.

The (commutative) action of dissipation and free evolution (7) on the covariance matrix is described by the map $\mathcal{E}_{\text {th }, \phi}(\sigma)=e^{-\gamma t} \mathcal{R}_{\phi} \sigma \mathcal{R}_{\phi}{ }^{T}+\left(1-e^{-\gamma t}\right) \sigma_{\text {th }}$, where $\sigma_{\text {th }}=(\bar{n}+$ $\left.\frac{1}{2}\right) \mathbb{1}_{2}$ is the covariance matrix of a thermal state and $\mathcal{R}_{\phi}$ is the rotation matrix due to harmonic evolution. Equivalently, under $\mathcal{E}_{\text {th, } \phi}$, the input state gets rotated and mixed with a thermal state via a beam splitter of effective transmissivity $\eta=e^{-\gamma t}$.

Measurement and dissipation compete over time. The former tries to reduce the uncertainty in one quadrature (at the expense of the other), while the latter tries to restore isotropy. Crucially, the spacing between two pulses determines the amount of mixing between the quadratures from one measurement to the next one. This consideration applies to any sequence of equally spaced pulses; for example, one can obtain a recursion relation $\sigma^{(N)}=\left(\mathcal{E}_{\Upsilon} \circ \mathcal{E}_{\text {th }, \phi}\right) \sigma^{(N-1)}$ to model a short train of pulses. This operation regime has recently become experimentally relevant for quantum applications [23]. Here we focus on a different regime: when the action of the measurement is undone by the dissipation there is no net effect over a unit cell and the system reaches a stroboscopic steady state [see Fig. 1(d)]. More formally, this state is a fixed point of the map $\mathcal{E}_{\Upsilon} \circ \mathcal{E}_{\text {th }, \phi}$; namely, it satisfies $\sigma_{s s}=\left(\mathcal{E}_{\Upsilon} \circ\right.$ $\left.\mathcal{E}_{\mathrm{th}, \phi}\right) \sigma_{s s}$. We stress that the two operations do not commute, so that in general $\mathcal{E}_{\Upsilon} \circ \mathcal{E}_{\text {th, } \phi} \neq \mathcal{E}_{\text {th }, \phi} \circ \mathcal{E}_{\Upsilon}$, as we shall see below. This is a novel regime for cavity optomechanics, which has focused either on steady-state properties of continuously driven systems or in the finite-time dynamics, as in pulsed optomechanics.

\section{STROBOSCOPIC SQUEEZING OF MECHANICAL MOTION}

\section{A. Stroboscopic backaction-evading measurement}

The first case we consider is that of a stroboscopic BAE measurement, for which a classical treatment is discussed in Refs. [26,31,32]. By choosing pulses interspaced by a multiple of half the mechanical period, we can in principle realize a QND measurement of position. Indeed, one has $[\hat{x}(t), \hat{x}(t+T)]=i \sin \left(\omega_{m} T\right)$, so that at the stroboscopic times $T=k \pi / \omega_{m}$ a sequence of precise position measurements is possible with no fundamental limit imposed by quantum mechanics (in the following we always take the shortest interval $k=1$ ). However, due to the presence of the environment, the covariance matrix does not come back to itself half a period later. We then look for solutions where the combined action of the measurement and the environment leaves the state invariant. Solving for the stroboscopic steady state $\sigma_{s s}=\left(\mathcal{E}_{\Upsilon} \circ \mathcal{E}_{\mathrm{th}, \pi}\right) \sigma_{s s}$, we get

$$
\begin{gathered}
\sigma_{X_{m}}=\frac{2 \bar{n}+1}{1+z+\sqrt{1+z^{2}+2 z \operatorname{coth}\left(\frac{\gamma T}{2}\right)}}, \\
\sigma_{P_{m}}=\bar{n}+\frac{1}{2}+\frac{\chi^{2}}{2\left(1-e^{-\gamma T}\right)}
\end{gathered}
$$



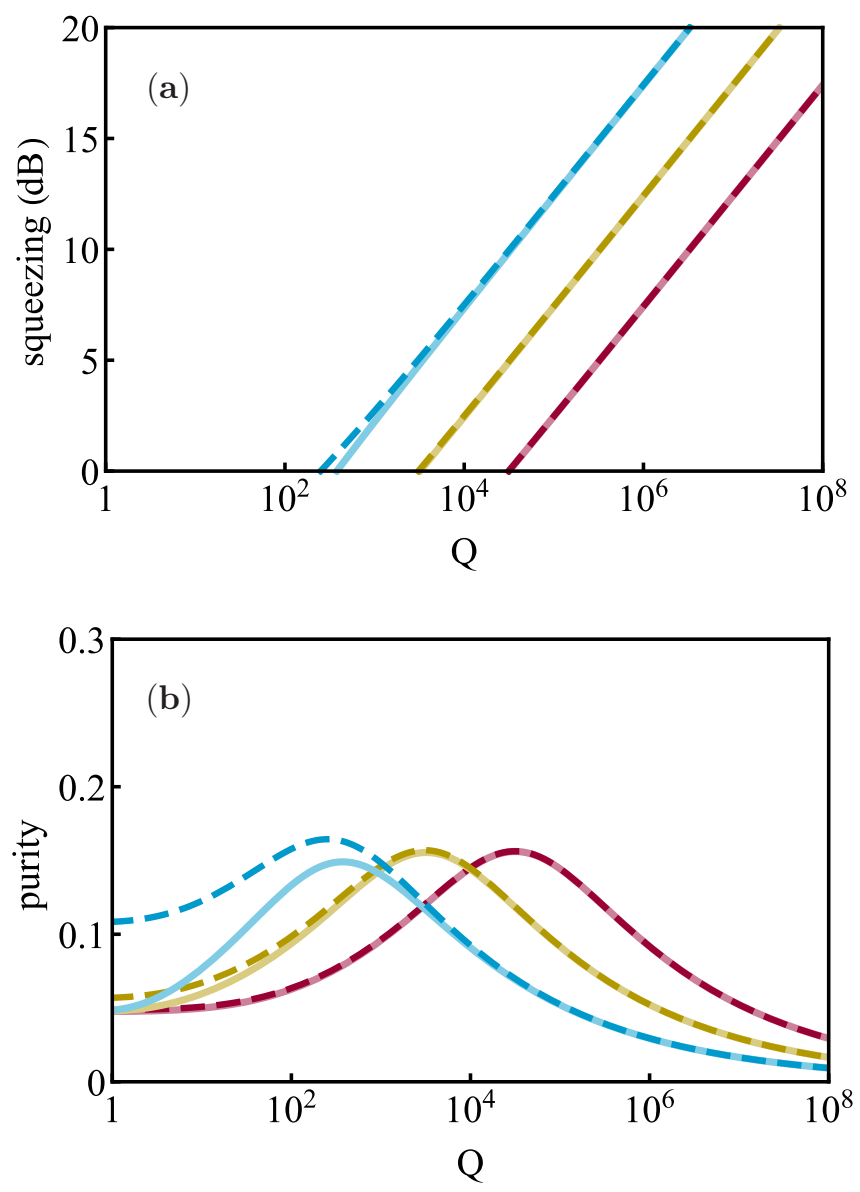

FIG. 2. Steady-state value of (a) mechanical squeezing and (b) mechanical purity for a stroboscopic BAE measurement. Values of the coupling are $\chi=0.05$ (red), $\chi=0.1$ (yellow), $\chi=0.5$ (cyan). Solid lines are for the stroboscopic steady state of the map $\mathcal{E}_{\text {th }} \circ \mathcal{E}_{\Upsilon}$, while dashed lines are for $\mathcal{E}_{\Upsilon} \circ \mathcal{E}_{\text {th }}$. The mean phonon number is set to $\bar{n}=10$.

and $\sigma_{X_{m} P_{m}} \equiv 0$, where we set $z=(2 \bar{n}+1) \chi^{2}$. We stress that the knowledge of such state is conditioned on the stream of measurement results. These expressions can be considerably simplified for large values of the mechanical quality factor $Q=\omega_{m} / \gamma$. The leading terms in the expansion are given by

$$
\sigma_{X_{m}}=\frac{\sqrt{2 \pi(\bar{n}+1 / 2)}}{2 \chi \sqrt{Q}}, \quad \sigma_{P_{m}}=\bar{n}+\frac{1}{2}+\frac{Q \chi^{2}}{2 \pi} .
$$

This simple result provides a quantum-mechanical treatment of stroboscopic BAE measurement and proves that mechanical decoherence does not preclude the occurrence of squeezing at long times. Indeed, uncertainty may fall below the zeropoint value, which implies a squeezed state of the resonator. Mechanical squeezing [expressed in $-10 \log _{10}\left(2 \sigma_{X_{m}}\right)$ decibel (dB)] is plotted Fig. 2(a). We stress that different $Q$ entail different characteristic times to approach the stroboscopic steady state.

The solid lines are for the steady state relative to $\mathcal{E}_{\mathrm{th}, \pi} \circ \mathcal{E}_{\Upsilon}$, while the dashed lines are for $\mathcal{E}_{\Upsilon} \circ \mathcal{E}_{\mathrm{th}, \pi}$. Physically, they correspond to knowledge of the conditional state directly after or directly before the measurement. We can see discrepancies arising due to the noncommutative character of the two maps for low $Q$ and large coupling values. In this parameter regime, if we start from a thermal state, the effects of measuring first are (partially) undone by the subsequent application of the thermal channel. On the other hand, by reversing the order (i.e., considering the map $\left.\mathcal{E}_{\Upsilon} \circ \mathcal{E}_{\mathrm{th}, \pi}\right) \mathcal{E}_{\mathrm{th}, \pi}$ acts as the identity, so the first measurement retains more conditioning power. The difference between the two cases thus boils down to an extra pulse, which has significative impact for large $\chi$ and explains the larger amount of squeezing. However, already for moderately large quality factors the two predictions coincide.

It is interesting to compare the condition for mechanical squeezing enforced by Eq. (13) with that required by pulsed optomechanics, i.e., by applying a single pulse (5). For a single pulse, values of the coupling $\chi>1$ are required to obtain squeezing (independently of $\bar{n}$ ), which has so far precluded reaching the quantum regime in pulsed optomechanics experiments. On the other hand, with stroboscopic driving approaching $\sigma_{X_{m}}<1 / 2$ only requires $\chi>\sqrt{2 \pi(\bar{n}+1 / 2) / Q}$, which can be considerably less demanding for large quality factors. In terms of the multiphoton quantum cooperativity $\mathcal{C}_{q}$ [1], the above requirement reads $\mathcal{C}_{q}>8 \pi /\left(\kappa \omega_{m} \tau^{2}\right)$.

Finally, if we compare how the two variances in Eq. (13) scale with $Q$, it is clear that fluctuations increase faster in $\hat{P}_{m}$ than they are reduced along $\hat{X}_{m}$. This means that, while getting squeezed, the resonator also gets heated up. This fact is highlighted in Fig. 2(b) where the mechanical purity $\mu=$ $\operatorname{Tr}\left[\hat{\varrho}_{s s}^{2}\right]$ for the same cases of Fig. 2(a) is shown. In the large- $Q$ limit the purity takes the simple form

$$
\mu=\pi^{1 / 4}\left(\frac{Q}{2 \bar{n}+1}\right)^{1 / 4} \sqrt{\frac{\chi}{2 \pi \bar{n}+Q \chi^{2}}} .
$$

Larger values of squeezing are accompanied by low purity. We will see in Sec. V that this conclusion gets drastically modified by considering the imperfect QND regime determined by mechanical evolution during the pulse.

\section{B. Squeezed input pulses}

Finally, we notice that the former results can be extended to the case where squeezed pulses, rather than coherent ones, are fed to the optomechanical cavity. In our simple model this observation amounts to replacing the cavity vacuum seed state $\sigma_{\text {pulse }}=\mathbb{1} / 2$ [see Eq. (4)] with a squeezed state $\sigma_{\text {pulse }}=\operatorname{diag}\left(\frac{e^{r}}{2}, \frac{e^{-r}}{2}\right)$ squeezed along the phase quadrature. This determines reduced fluctuations of the (measured) optical phase, which in turn enhances the conditioning effect of the measurement. One obtains results as in Eqs. (11) and (12) with the substitution $\chi \rightarrow e^{\frac{r}{2}} \chi$; namely, a train of squeezed pulses magnifies the measurement strength by an exponential factor (in the degree of squeezing).

\section{STROBOSCOPIC GROUND-STATE COOLING}

Another interesting case is obtained by spacing the pulses by a quarter of a period. In this case the value of the variance along $\hat{X}_{m}$ and $\hat{P}_{m}$ gets swapped by the free evolution, so that the measurement reduces both variances alternately. An exact expression for the stroboscopic steady state is available also in 


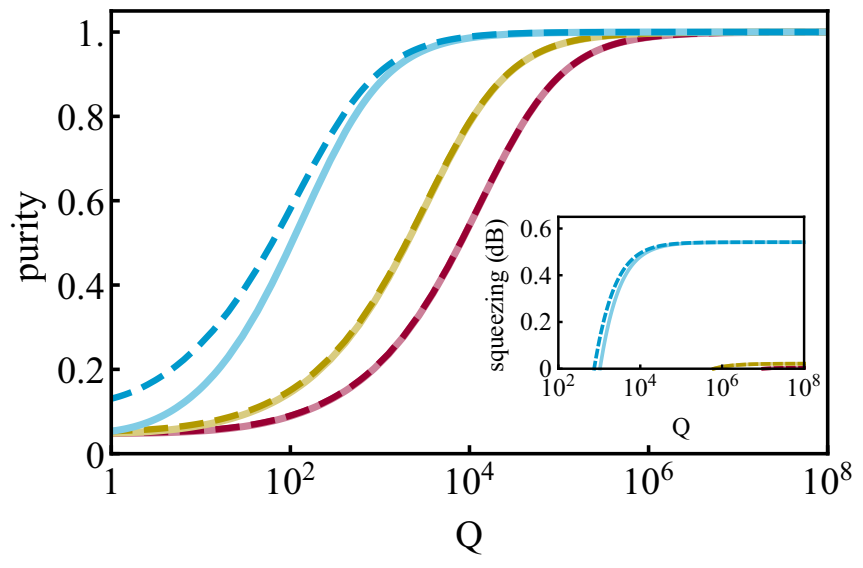

FIG. 3. Stroboscopic ground-state cooling. Steady-state value of the mechanical purity for pulses spaced by a quarter of the mechanical period. Values of the coupling are $\chi=0.05$ (red), $\chi=0.1$ (yellow), $\chi=0.5$ (cyan). Solid lines are for the stroboscopic steady state relative to the map $\mathcal{E}_{\text {th }} \circ \mathcal{E}_{\Upsilon}$, while dashed lines are for the operations applied in reversed order. The mean phonon number is set to $\bar{n}=10$. In the inset we show residual asymmetry between the two quadratures [cf. Eqs. (15) and (16)], which can result in a small amount of squeezing.

this case, although quite cumbersome. For convenience below we give the expansion for large $Q$ :

$$
\begin{aligned}
\sigma_{X_{m}} & =\frac{\sqrt{4+\chi^{4}}-\chi^{2}}{4}+\frac{\mathcal{F}(\chi, \bar{n})}{Q}, \\
\sigma_{P_{m}} & =\frac{\sqrt{4+\chi^{4}}+\chi^{2}}{4}+\frac{\mathcal{G}(\chi, \bar{n})}{Q} .
\end{aligned}
$$

The full expression of the functions $\mathcal{F}(\chi, \bar{n}), \mathcal{G}(\chi, \bar{n})$ is reported in Appendix B. Unlike Eq. (13), now fluctuations in both quadratures converge to a constant value for $Q \rightarrow \infty$. We also notice that there is a residual asymmetry between the two quadratures. The leading terms therefore describe a squeezed vacuum state, albeit one where the squeezing grows slowly with the coupling $\chi$. For realistic values of the coupling the state is thus only weakly squeezed and has near-unit fidelity with the mechanical vacuum. The conditional purification of the mechanical state is also known as cooling by measurement [22]. In the same spirit, we refer to this case as stroboscopic cooling. Of course for finite values of the quality factor the steady state will be mixed, but cooling close to the ground state is still possible. We show these features in Fig. 3.

\section{IMPROVED DESCRIPTION AND NUMERICAL SIMULATIONS}

In this section we aim to provide a more accurate description of the stroboscopic conditional dynamics. We focus on the case of stroboscopic BAE measurements but the analysis can be readily extended to the case of stroboscopic cooling. We expand along two directions: (i) we model the measurement as actually taking place outside the optical cavity and (ii) we evaluate the effects of the mechanical free evolution during the pulsed interaction. To this end, we consider the following extended Hamiltonian:

$$
\hat{H}=\hat{H}_{I}(t)+i \sqrt{\kappa}\left(\hat{a}^{\dagger} \hat{a}_{\mathrm{in}, t}-\hat{a} \hat{a}_{\mathrm{in}, t}^{\dagger}\right),
$$

where, beside the term in Eq. (1), we also include an interaction with the continuum of electromagnetic modes $\hat{a}_{\text {in }, t}$ living outside the cavity. This stream of modes interacts with the system at time $t$ and is otherwise uncorrelated $\left[\hat{a}_{\mathrm{in}, t}, \hat{a}_{\mathrm{in}, t^{\prime}}^{\dagger}\right]=$ $\delta\left(t-t^{\prime}\right)$. As is customary, we assume they have the Markovian correlation function $\left\langle\left\{\hat{a}_{\mathrm{in}, t}, \hat{a}_{\mathrm{in}, t^{\prime}}^{\dagger}\right\}\right\rangle=\delta\left(t-t^{\prime}\right)$.

For a short pulse of length $\tau$ (for now neglecting the free mechanical evolution) the corresponding propagator takes the form

$$
\hat{U}=e^{i \chi \hat{X}_{m} \hat{X}_{c}+i \sqrt{\kappa \tau}\left(\hat{P}_{c} \hat{X}_{\mathrm{in}}-\hat{X}_{c} \hat{P}_{\mathrm{in}}\right)}
$$

where $\hat{X}_{\text {in }}, \hat{P}_{\text {in }}$ are the proper (dimensionless) modes of the environment, i.e., $\left[\hat{X}_{\text {in }}, \hat{P}_{\text {in }}\right]=i$, which are being measured; homodyne detection of the phase quadrature corresponds to projection along $\left|P_{\text {in }}\right\rangle$ (see Appendix C for details).

Formally, we can then proceed as in Sec. II to compute the conditional covariance matrix of the optomechanical system, include thermal decoherence, and enforce the stroboscopic steady-state condition. The full expression of the conditional state of the mechanical system is quite cumbersome, but in the large- $Q$ limit we get the following simple expressions

$$
\begin{aligned}
\sigma_{X_{m}} & =\frac{\kappa \sqrt{2 \pi(\bar{n}+1 / 2)}}{4 g \sin ^{2}\left(\frac{\sqrt{\kappa \tau}}{2}\right) \sqrt{\kappa \tau Q}}, \\
\sigma_{P_{m}} & =\bar{n}+\frac{1}{2}+\frac{g^{2} Q \tau[1-\cos (\sqrt{\kappa \tau})]}{\pi \kappa},
\end{aligned}
$$

with $g=2 g_{0} \sqrt{N_{\mathrm{p}} /(\kappa \tau)}$. These expressions are to be seen as a refinement of Eq. (13); as we will show, they offer a useful comparison with numerical simulations.

Second, we include corrections to the ideal QND limit stemming from the mixing of the mechanical quadratures during a pulse of finite length. The ensuing unitary evolution contains two new terms (see Appendix A for the full expression): a squeezing term in the cavity amplitude, which however is $O\left(g^{2} / \omega_{m}^{2}\right)$, and a spurious term $\propto \hat{X}_{c} \hat{P}_{m}$, which spoils the QND nature of the interaction. The strength of this term is $2 \omega_{m} / \kappa$ times the QND part, so that the QND limit is approximately recovered only for optomechanical systems deep in the bad-cavity regime. It is therefore important to address the corrections arising for finite values of the sideband parameter, which limit the amount of conditional squeezing attainable.

Due to the presence of quadrature mixing, a closed expression of the conditional state can no longer be found. However, we can get a clear physical picture of the effects brought about by non-QND terms in the following way. Consider the effective Hamiltonian generating the optomechanical evolution, first neglecting and then including the non-QND term (to faithfully model the measurement, we also include the interaction with the extra-cavity modes); the corresponding expressions are given by Eqs. (1) and (A6), respectively. We can use them to compute the Heisenberg evolution of the quadratures in both cases, $\dot{\hat{X}}_{m}=\ldots, \dot{\hat{P}}_{m}=\ldots$, and so on, where the terms appearing on the right-hand side drive the evolution of a given quadrature. The equations of motion 

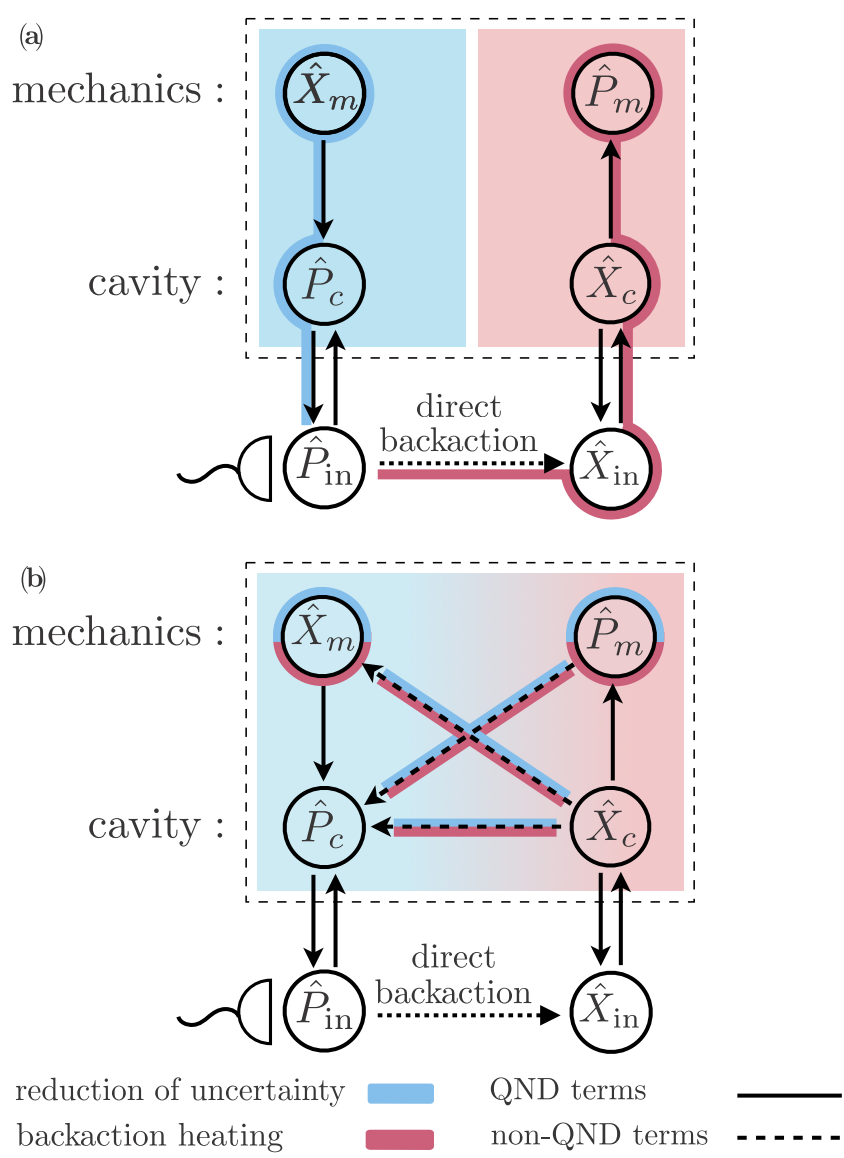

FIG. 4. Effects of the measurement for (a) an ideal QND interaction and (b) including mechanical free evolution during each pulse. The black arrows describe how quadratures influence each other in an ideal QND interaction [evolution according to Eq. (18)]. Measurement of the output phase quadrature both introduces backaction and allows to extract information. (a) In an ideal stroboscopic measurement, measurement backaction and reduction of uncertainty take two distinct paths (the first is confined to $\hat{P}_{m}$ while the second to $\hat{X}_{m}$ ) which allows squeezing along $\hat{X}_{m}$. (b) Non-QND terms open new paths (dashed arrows) where both conditioning and backaction spread. These reduce squeezing in $\hat{X}_{m}$, but at the same time enable acquiring information about all the quadratures, which results in larger mechanical purities and optomechanical entanglement.

for the two cases are schematized in Figs. 4(a) and 4(b), respectively, where an arrow connecting two terms means that the variable at the starting point drives the evolution of that at the ending point. Next, we incorporate the role of the measurement, which has a twofold effect: on the one hand, it enables the acquisition of information, i.e., reducing the uncertainty about the mechanical quadrature $\hat{X}_{m}$; this acquisition happens indirectly through the optomechanical coupling and requires that we keep track of the stochastic component. On the other hand, the measurement introduces disturbance, which directly affects the conjugate quadrature $\left(\hat{X}_{\text {in }}\right)$ and then, through the dynamics, reaches the mechanical system.

In the ideal QND case [see Fig. 4(a)] these two effects fully decouple. Fluctuations are reduced along $\hat{X}_{m}$ and increased in $\hat{P}_{m}$ (backaction heating). Graphically, this corresponds to the fact that no arrow points toward $\hat{X}_{m}$, and hence no noise can drive it. Likewise, no arrow originates from $\hat{P}_{m}$, which "absorbs" all the backaction. Thus backaction confinement enables repeated measurements of the same quadrature with no added noise, which is the working principle of BAE measurements. When we take into account the finite mechanical evolution [cf. Fig. 4(b)], the non-QND terms open new paths (dashed arrows) for both backaction and conditioning to spread, with the following consequences: information is now acquired about both mechanical quadratures (and hence fluctuations of the conditional state are reduced in both directions) which entails that (i) the measurement purifies the state. Similarly, measurement backaction is no longer confined to $\hat{P}_{m}$ but extends to both quadratures, i.e., (ii) the amount of squeezing is reduced with respect to the ideal case. Finally, information is simultaneously acquired about both the cavity and the mechanics (see multiple arrows incoming at $\hat{P}_{c}$ ); such joint reduction of the uncertainty implies that (iii) correlations between cavity and mechanics are built. Depending on the occupancy of the mechanical bath, this may even lead to entanglement being established between the two resonators. We want to remark that, while the limitation (ii) posed by non-QND terms is known, their beneficial effects (i) and (iii) have not been previously appreciated. A similar situation is encountered in continuous BAE measurements, where RWA solution yields conditional squeezing with low purity, and the inclusion of counter-rotating terms lower the amount of squeezing but at the same time allows for larger purity and optomechanical entanglement [39].

To check the validity of these conclusions we numerically integrate the conditional evolution of the full optomechanical system subject to stroboscopic driving and continuous homodyne detection of the output phase quadrature (see Refs. [36,39] for details). Free mechanical evolution during each pulse is explicitly included in the simulation, i.e., we use the optomechanical interaction in Eq. (1). In Fig. 5(a) we show the numerical squeezing in the long-time limit (averaged over one period) and compare it with the prediction of Eq. (19). Our simple analytical formula shows excellent agreement except for large $Q$, where it does not capture the saturation of squeezing. Such saturation confirms our expectation (ii). Indeed, for a fixed duration of the pulse, the effects of the free mechanical evolution become more prominent for larger $Q$. From Fig. 5(b) we see that a realistic stroboscopic BAE measurement actually generates highly pure conditional squeezed states. Mixing of the two quadratures, present for any finite value of the sideband parameter, implies the simultaneous squeezing and cooling the mechanics by stroboscopic BAE measurement, as predicted in (i). Finally, in Fig. 5(c) the conditional optomechanical entanglement is displayed (quantified by the logarithmic negativity) which confirms (iii). Notice that entanglement is present in the high-temperature regime.

\section{VERIFICATION OF THE MECHANICAL STATE: STROBOSCOPIC TOMOGRAPHY}

Essential to any conditional protocol is a verification part. While in the previous sections we have focused on state preparation through measurement, here we calculate how well 

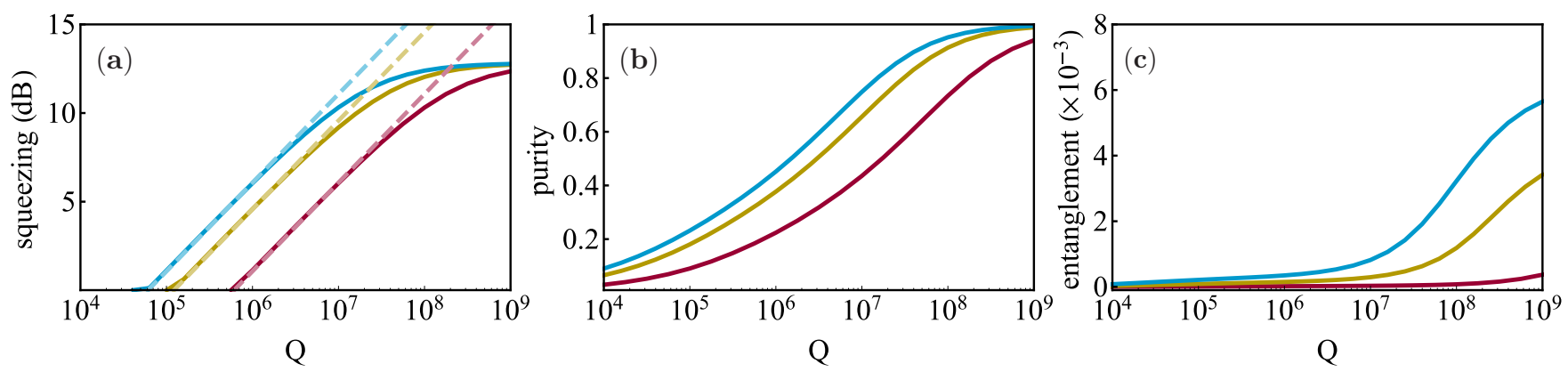

FIG. 5. (a) Mechanical squeezing in a stroboscopic BAE measurement. Solid lines are for the numerical solution and dashed lines are for the prediction based on Eqs. (19) and (20). The curves are for different strengths, parametrized by the number of photons $N_{\mathrm{p}}=10^{6}$ (red), $N_{\mathrm{p}}=$ $5 \times 10^{6}$ (yellow), and $N_{\mathrm{p}}=10^{7}$ (cyan). Other parameters are $g_{0}=5 \times 10^{-4} \omega_{m}, \bar{n}=1000, \eta=1, \kappa=15 \omega_{m}, \tau=0.3 \omega_{m}^{-1}$. (b) Mechanical purity and (c) entanglement (measured by the logarithmic negativity) between optical cavity and mechanical resonator for the same set of parameters.

a quadrature can be measured in a train of pulses. This is also known as retrodiction [40,41]. State verification via retrodiction has been recently employed to verify the quantum trajectory of a continuously driven optomechanical system [42]. The final result of a stroboscopic measurement is a measurement value with a given confidence interval. Repeatedly preparing and measuring a state allows for full tomography. Since it makes sense to keep measuring until the resonator is no longer correlated with its initial state, the resonator state at the end of the measurement is again a conditionally squeezed state as discussed above, independent of the initial state.

In a stroboscopic measurement of a harmonic oscillator, its position is measured at regular intervals. This results in a string of measurement results $\vec{y}=\left(y_{0}, y_{1}, y_{2}, \ldots\right)$, which are correlated with the actual position at that time $y_{i}=x_{i}+$ $m_{i}$. The measurement errors $\left\{m_{i}\right\}$ are normally distributed as the Kraus operator corresponding to the measurement (5) predicts. Specifically, if the oscillator is in a position eigenstate $\rho=|x\rangle\langle x|$, the measurement probability distribution is $p\left(P_{c}\right)=\operatorname{Tr}\left[\hat{\Upsilon}^{\dagger} \hat{\Upsilon} \rho\right] \propto \exp \left[-\left(P_{c} / \chi-x\right)^{2} / \chi^{-2}\right]$. Thus, the $\left\{m_{i}\right\}$ are drawn from a Gaussian distribution of zero mean, with variance $\sigma_{m}^{2}=1 /\left(2 \chi^{2}\right)$. To realize a QND measurement, the time $t_{n}$ between measurements has to be an integer multiple of half a period, $t_{n}=(n+1) \pi / \omega_{m}$. This is because $\left[\hat{x}\left(t_{n}\right), \hat{x}\left(t_{m}\right)\right]=0$, i.e., mechanical position becomes a QND observable at these stroboscopic times. In this regime, the problem becomes classical, as the measurement backaction is evaded. Here we choose the time between measurements to be as short as possible, $T=\pi / \omega_{m}$, such that $\gamma T=\pi / Q$. In between each measurement, the position of the oscillator decays, due to damping, and gets a random contribution from the thermal noise acting, $x_{i}=e^{-\gamma T / 2} x_{i-1}+d_{i}$. The random numbers $d_{i}$ also follow a normal distribution of mean zero and standard deviation $\sigma_{d}^{2}=(\bar{n}+1 / 2)\left(1-e^{-\gamma T}\right)$ which follows from the fluctuation-dissipation theorem (or equivalently from the explicit discussion in Sec. II).

In Appendix D we show that, given a string of measurement results $\vec{y}$, the conditional probability distribution inferred from Bayes' theorem is the normal distribution

$$
\vec{x} \sim N\left(\vec{\mu}_{x}+\mathrm{Q}_{x x}^{-1} \vec{y} / \sigma_{m}^{2}, \mathrm{Q}_{x x}^{-1}\right),
$$

where the correlation matrix $\mathrm{Q}_{x x}$ is given by

$$
\begin{aligned}
{\left[\mathrm{Q}_{x x}\right]_{11} } & =\frac{1}{\sigma_{x_{0}}^{2}}+\frac{1}{\sigma_{m}^{2}}+\frac{e^{-\gamma T}}{\sigma_{d}^{2}}, \\
{\left[\mathrm{Q}_{x x}\right]_{i, i \pm 1} } & =-\frac{e^{-\gamma T / 2}}{\sigma_{d}^{2}}, \\
{\left[\mathrm{Q}_{x x}\right]_{i i} } & =\frac{1}{\sigma_{m}^{2}}+\frac{1+e^{-\gamma T}}{\sigma_{d}^{2}}, \\
{\left[\mathrm{Q}_{x x}\right]_{n n} } & =\frac{1}{\sigma_{m}^{2}}+\frac{1}{\sigma_{d}^{2}} .
\end{aligned}
$$

Interestingly, as the number of measurements goes to infinity, the matrix $Q_{x x}$ can be inverted analytically (see Appendix D2). The first element of the inverse, $\left[Q_{x x}^{-1}\right]_{11}$, is the variance associated with the measured value, and as one would expect it coincides with the achieved squeezing [Eq. (13)]. While perhaps this could have been inferred from the results above, a very good approximation to the inverse and thus the variance can also be found for a finite number of measurements. Furthermore, this approach yields the weight each measurement value is associated with, although the general expression is somewhat unenlightening (see Appendix D). For the experimentally relevant case of small $\bar{n} / Q$, measurements are weighted by exponentially reducing factors with increasingly distant measurement times [see Eq. (D17)]. Finally, we can also show that, when taking into account many measurements before and after a certain point in time, i.e., using preparation and retrodiction, the associated variance is half of Eq. (13).

\section{COLLECTIVE ENTANGLEMENT AND COOLING OF TWO MECHANICAL RESONATORS}

In this section, we show how the previous results can be extended to the case of two nondegenerate mechanical resonators. To do that, we consider two mechanical resonators of frequency $\omega_{m, 1}$ and $\omega_{m, 2}$ coupled to a common cavity field. Collective BAE schemes have been proposed in this configuration for continuous and two-tone driving [43-45]. We introduce the mean and the relative mechanical frequency, respectively defined as $\omega=\left(\omega_{m, 1}+\omega_{m, 2}\right) / 2$ and $\Omega=\left(\omega_{m, 1}-\right.$ $\left.\omega_{m, 2}\right) / 2$ (we assume $\omega_{m, 1}>\omega_{m, 2}$ without loss of generality). 
We also define the collective mechanical variables

$$
\hat{X}_{ \pm}=\left(\hat{X}_{m, 1} \pm \hat{X}_{m, 2}\right) / \sqrt{2}, \quad \hat{P}_{ \pm}=\left(\hat{P}_{m, 1} \pm \hat{P}_{m, 2}\right) / \sqrt{2},
$$

which satisfy $\left[\hat{X}_{ \pm}, \hat{P}_{ \pm}\right]=i,\left[\hat{X}_{ \pm}, \hat{P}_{\mp}\right]=0$. When the pulse is on, both mechanical resonators linearly couple to the common cavity amplitude, giving

$$
\begin{aligned}
\hat{H}_{I}(t) & =-g(t) \sum_{j=1,2} \hat{X}_{c}\left[\hat{X}_{m, j} \cos \left(\omega_{m, j} t\right)+\hat{P}_{m, j} \sin \left(\omega_{m, j} t\right)\right] \\
& =-\sqrt{2} g(t) \hat{X}_{c}(\hat{X} \cos \omega t+\hat{Y} \sin \omega t) .
\end{aligned}
$$

For simplicity, we have considered the case of equal singlephoton optomechanical couplings and in the second line we have rewritten the interaction in terms of the rotated collective quadratures

$$
\begin{aligned}
& \hat{X}=\hat{X}_{+} \cos \Omega t+\hat{P}_{-} \sin \Omega t, \\
& \hat{Y}=\hat{P}_{+} \cos \Omega t-\hat{X}_{-} \sin \Omega t,
\end{aligned}
$$

which still form a conjugate pair $[\hat{X}, \hat{Y}]=i$. Thanks to this change of variables we see that Eq. (25) has the same form as Eq. (1) and therefore we can rely on our previous analysis. In particular, for $g(t)=g \delta(t-k \pi / \omega)$ we recover the ideal case of stroboscopic QND interaction $\hat{U} \approx e^{i \sqrt{2} \chi \hat{X}_{c} \hat{X}}$ (here $k=1$ ). This corresponds to pulsing every half of the fundamental period $2 T_{1} T_{2} /\left(T_{1}+T_{2}\right)$, where $T_{j}$ are the single mechanical periods. Like in the single-mode case, we also include mechanical dissipation. For simplicity, in the following we consider equal mechanical damping rates and the same occupancies for the two baths. For nondegenerate mechanical modes, these conditions may entail adjusting the local temperatures of the baths to achieve the same occupancy.

From the discussion of Sec. III A we conclude that the stroboscopic steady state is a squeezed thermal state in the collective variables $\hat{X}$ and $\hat{Y}$, with the variance reduced along $\hat{X}$ and heated up along $\hat{Y}$ by the backaction. We now want to express the state in terms of the original local variables. For this purpose, it is useful to parametrize the steady state $\sigma_{s s}$ of single-mode BAE measurements [cf. Eqs. (11) and (12)] as

$$
\sigma_{s s}=\operatorname{diag}\left[\left(n_{\mathrm{eff}}+\frac{1}{2}\right) e^{-r_{\mathrm{eff}}},\left(n_{\mathrm{eff}}+\frac{1}{2}\right) e^{r_{\mathrm{eff}}}\right],
$$

where an explicit expression of $n_{\text {eff }}, r_{\text {eff }}$ can be obtained by inverting Eqs. (11) and (12) (one also needs to rescale $g \rightarrow$ $g / \sqrt{2})$. Next, we notice that the vector of original quadratures $\hat{Q}=\left(\hat{X}_{m, 1}, \hat{P}_{m, 1}, \hat{X}_{m, 2}, \hat{P}_{m, 2}\right)^{T}$ and that of collective ones $\hat{Q}^{\prime}=(\hat{X}, \hat{Y}, \hat{W}, \hat{Z})^{T}$ are related via the following transformation: $\quad \hat{Q}^{\prime}=\hat{U}_{B S}^{\dagger} e^{i \Omega t\left(\hat{b}_{1}^{\dagger} \hat{b}_{1}-\hat{b}_{2}^{\dagger} \hat{b}_{2}\right)} \hat{Q} e^{-i \Omega t\left(\hat{b}_{1}^{\dagger} \hat{b}_{1}-\hat{b}_{2}^{\dagger} \hat{b}_{2}\right)} \hat{U}_{B S}$; here $\hat{Z}=\hat{X}_{-} \cos \Omega t+\hat{P}_{+} \sin \Omega t$ and $\hat{W}=\hat{P}_{-} \cos \Omega t-$ $\hat{X}_{+} \sin \Omega t$ are the other two collective rotated quadratures and $\hat{U}_{B S}$ is a beam-splitter transformation. Note that the modes are rotated in opposite directions before getting mixed. By transforming state (27) accordingly we get

$$
\sigma_{s s}=\left(\begin{array}{cc}
A & C \\
C^{T} & A
\end{array}\right),
$$

where $\quad A=\left(n_{\mathrm{eff}}+\frac{1}{2}\right) \cosh 2 r_{\mathrm{eff}} \mathbb{1}_{2} \quad$ and $\quad C=-\left(n_{\mathrm{eff}}+\right.$ $\left.\frac{1}{2}\right) \sinh 2 r_{\text {eff }} \sigma_{z}$ (with $\sigma_{z}$ being the $z$ Pauli matrix), namely, a two-mode squeezed thermal state. This state is known to be entangled if and only if $r_{\text {eff }}>\ln \left(\sqrt{1+2 n_{\text {eff }}}\right)$. Therefore, frequent measurements modeled by the pulses may induce entanglement between the two noninteracting resonators.

The same argument can be repeated for two-mode cooling, which corresponds to pulsing every quarter of the fundamental period $2 T_{1} T_{2} /\left(T_{1}+T_{2}\right)$. Indeed, Eqs. (15) and (16) are also in the form of a squeezed thermal state, although with very little squeezing. Therefore the two-mode stroboscopic steady state is still of the form (28), the difference being that now we have $n_{\mathrm{eff}} \approx 0$ and smaller $r_{\mathrm{eff}}$ (compared with the previous case). For both $n_{\mathrm{eff}}, r_{\mathrm{eff}} \approx 0$ the steady state has a large overlap with the vacuum of the two modes. Notice however that for $n_{\mathrm{eff}}=0$ the state is entangled for any value of $r_{\text {eff }}>0$.

\section{EXPERIMENTAL CONSIDERATIONS}

The principal considerations for implementing stroboscopic optomechanics concern the appropriate hierarchy of timescales $\kappa^{-1} \ll \tau \ll \omega_{m}^{-1}$ and sufficient measurement strength $\chi$. Low bath occupation $\bar{n}$ and high quality factors $Q$ facilitate access to the quantum regime. However, experimental nonidealities have to be taken into account as well. These can include subunity detection efficiency, optical absorption heating, mechanical frequency drift, and spurious mechanical modes, among others.

Given the recent progress with measurement-based quantum-state preparation [42,46] with membrane-in-themiddle optomechanical systems, we discuss this platform first. With $\mathrm{MHz}$ resonance frequencies, sufficiently short (submicrosecond) pulses are readily implemented using standard modulation techniques. Such pulses could be accommodated in short $(L \approx 1 \mathrm{~mm})$, medium-finesse resonators with $\kappa / 2 \pi \gtrsim 15 \mathrm{MHz}$, and the cavity output detected with high efficiency, as previously demonstrated $[42,46]$. Optical power levels tolerated in continuous-wave operation [46] suggest $\chi=g \tau \approx 0.1$ can be achieved without significant device heating at a temperature of $\approx 10 \mathrm{~K}$, or $\bar{n} \approx 10^{5}$. Since stroboscopic operation would lower the thermal load from optical absorption by a factor of order $\omega_{m} \tau<1$, even higher $\chi$ may be possible, provided instabilities are avoided and the cavity lock maintained. If the experiment were implemented with soft-clamped membrane resonators [42,46,47], very high quality factors $Q \approx 10^{9}$ are available (for comparison with some of the results presented so far, gentle laser precooling can be assumed to trade equivalent bath occupancy with quality factor, leaving the ratio $\bar{n} / Q$ constant). With this set of parameters, significant levels of squeezing can be achieved, see Fig. 5.

In practice, however, other mechanical modes at harmonics of the stroboscopic sampling frequency contribute to the measured signal, in an effect known as aliasing in the context of periodically sampled data. This would lead to spurious noise and interactions, and a degradation of the prepared state. In contrast with pulsed optomechanics [14], which offers virtually no spectral discrimination of mechanical modes at all, the stroboscopic protocols are only sensitive to spurious modes 
that coincide with a harmonic of the sampling frequency. Yet soft-clamped membrane resonators with their high density of states outside the band gap, in which the high- $Q$ modes lie, would be strongly affected by this effect. Membrane-in-themiddle setups with mechanical systems that feature a sparser mode spectrum, such as trampoline resonators $[48,49]$, may therefore be preferable, provided sufficiently high $Q$ factors and/or low temperatures can be achieved.

Nanophotonic structures could be an alternative platform of interest. Their already sparse mechanical mode spectrum could conceivably be engineered to be sufficiently anharmonic. Measurement strengths as high as $\chi \approx 0.1$ have already been demonstrated with single optical pulses much shorter $(\tau=20 \mathrm{~ns})$ than the mechanical period $\left(2 \pi / \omega_{m} \approx\right.$ $300 \mathrm{~ns}$ ) [23]. In combination with high-efficiency readout and efficient heat removal [50], stroboscopic optomechanics might also allow generation of squeezed and entangled states in such systems.

\section{CONCLUSIONS}

In this work we provided a description of the conditional dynamics of an optomechanical system driven by a train of pulses. We showed that the resulting frameworkdubbed stroboscopic quantum optomechanics-provides a versatile toolbox for measurement-based quantum control of optomechanical systems in the bad-cavity regime, ranging from ground-state cooling to mechanical squeezing, and applicable to single-mode as well as multimode optomechanics. Crucially, it enables the generation and characterization of measurement-based squeezing and entanglement. Stroboscopic driving alleviates the requirements of pulsed protocols based on a single (or a few) pulse(s).

\section{ACKNOWLEDGMENTS}

D.M. acknowledges support by the Horizon 2020 ERC Advanced Grant QUENOCOBA (grant agreement 742102). A.N. acknowledges a University Research Fellowship from the Royal Society and additional support from the Winton Programme for the Physics of Sustainability. A.S. acknowledges funding from the European Union's Horizon 2020 research and innovation program (European Research Council project Q-CEOM, Grant Agreement No. 638765). This work was supported by the European Union Horizon 2020 research and innovation program under Grant Agreement No. 732894 (FET Proactive HOT).

\section{APPENDIX A: DERIVATION OF THE PULSED INTERACTION}

In the following we derive the complete expression of the unitary evolution (2) induced by the pulsed interaction and discuss when the simple QND expression (3) is recovered. At a formal level, the evolution $\hat{U}\left(t, t_{0}\right)$ can be equivalently described by the Magnus expansion

$$
\hat{U}\left(t, t_{0}\right)=\exp \sum_{k=1}^{\infty} \hat{\Omega}_{k}\left(t, t_{0}\right),
$$

which comes in the form of a nonordered exponential. Compared with Eq. (2), the complexity of the expression has just been shifted to the argument of the exponential. The first two terms of the Magnus expansion are given by

$$
\begin{aligned}
& \hat{\Omega}_{1}\left(t, t_{0}\right)=-i \int_{t_{0}}^{t} d t_{1} \hat{H}_{I}\left(t_{1}\right), \\
& \hat{\Omega}_{2}\left(t, t_{0}\right)=-\frac{1}{2} \int_{t_{0}}^{t} d t_{1} \int_{t_{0}}^{t_{1}} d t_{2}\left[\hat{H}_{I}\left(t_{1}\right), \hat{H}_{I}\left(t_{2}\right)\right],
\end{aligned}
$$

where $\hat{H}_{I}(t)$ is the linearized interaction in the rotating frame, as given in Eq. (1). For concreteness, let us consider a rectangular pulse of length $\tau$ centered at the origin, described by the normalized profile $\varepsilon(t)=\frac{1}{\sqrt{\tau}}[\Theta(t-\tau / 2)-\Theta(t-\tau / 2)]$. When the pulse drives the optomechanical cavity on resonance, the evolution of the field amplitude inside the cavity is well approximated by $\dot{\alpha}=-\frac{\kappa}{2} \alpha+\sqrt{\kappa N_{\mathrm{p}}} \varepsilon(t)$, where we neglected the mechanical response during the short interaction time. The solution reads

$$
\alpha(t)=2 \sqrt{\frac{N_{\mathrm{p}}}{\kappa \tau}}\left[f_{+}(t) \Theta(t+\tau / 2)-f_{-}(t) \Theta(t-\tau / 2)\right],
$$

where we set $f_{ \pm}(t)=1-e^{-\frac{\kappa}{2}\left(t \pm \frac{\tau}{2}\right)}$. The expression captures the buildup and the decay of the coherent field inside the cavity. The time-dependent optomechanical coupling in Eq. (1) is $g(t)=g_{0} \alpha(t)$. Notice that, in the fast cavity limit, the expression reduces to a rectangular pulse of height $g_{\text {ad }}=$ $2 g_{0} \sqrt{N_{\mathrm{p}} /(\kappa \tau)}$.

We can now compute the expressions (A2a) and (A2b) for this profile. For late times $t \gg \tau$ we get

$$
\hat{\Omega}_{1}=i \chi \hat{X}_{c}\left(\hat{X}_{m}+\frac{2 \omega_{m}}{\kappa} \hat{P}_{m}\right), \quad \hat{\Omega}_{2}=i \frac{\zeta}{2} \hat{X}_{c}^{2},
$$

where the prefactors read

$$
\begin{aligned}
& \chi=2\left(\frac{g_{\mathrm{ad}}}{\omega_{m}}\right) \frac{\sin \left(\frac{\omega_{m} \tau}{2}\right)}{1+4\left(\frac{\omega_{m}}{\kappa}\right)^{2}}, \\
& \zeta=16\left(\frac{g_{\mathrm{ad}}}{\omega_{m}}\right)^{2} \frac{\sinh ^{2}\left(\frac{\kappa \tau}{4}\right)}{\left(\frac{\kappa}{\omega_{m}}\right)^{3}+4\left(\frac{\kappa}{\omega_{m}}\right)} .
\end{aligned}
$$

In the above expression we set $g_{\text {ad }}=2 g_{0} \sqrt{N_{\mathrm{p}} /(\kappa \tau)}$. In the adiabatic limit $\chi$ reduces to $\chi_{\mathrm{ad}}=g_{\mathrm{ad}} \tau \equiv 2 g_{0} \sqrt{N_{\mathrm{p}} \tau / \kappa}$, which is the expression given in the main text. Also notice that, as expected for the problem at hand, all the nested commutators corresponding to higher-order $\hat{\Omega}_{k \geqslant 3}$ identically vanish. The evolution thus takes the following exact form:

$$
\hat{U}=e^{i \frac{\zeta}{2} \hat{X}_{c}^{2}} e^{i \chi \hat{X}_{c} \hat{X}_{m}+i \chi\left(\frac{2 \omega_{m}}{\kappa}\right) \hat{X}_{c} \hat{P}_{m}} .
$$

Two extra terms have appeared compared with Eq. (3). A single-mode operator that is responsible for squeezing of the cavity field and an interaction term that spoils the QND character of the quadrature $\hat{X}_{m}$. Note that both terms are present for any length of the pulse, even though the spurious term is suppressed by a factor $2 \omega_{m} / \kappa$. The QND limit can then be recovered only for a vanishing sideband parameter $\omega_{m} / \kappa$. 


\section{APPENDIX B: COMPLETE EXPRESSION FOR STROBOSCOPIC COOLING}

The complete expressions of the mechanical variances at the stroboscopic steady state in the case of cooling [Eqs. (15) and (16)], up to $O\left(Q^{-1}\right)$, are given by

$$
\begin{aligned}
\sigma_{X_{m}} & =\frac{\sqrt{4+\chi^{4}}-\chi^{2}}{4}+\frac{\pi\left[-4 \bar{n} \chi^{2}+\frac{4 \bar{n}\left(\chi^{4}+2\right)+\chi^{6}+2 \chi^{4}+4 \chi^{2}+4}{\sqrt{\chi^{4}+4}}-\chi^{4}-2 \chi^{2}-2\right]}{8 Q \chi^{2}}, \\
\sigma_{P_{m}} & =\frac{\sqrt{4+\chi^{4}}+\chi^{2}}{4}+\frac{\pi\left[2 \bar{n}\left(\chi^{4}+2\right)+\chi^{4}-\sqrt{\chi^{4}+4}+2\right]}{4 Q \chi^{2} \sqrt{\chi^{4}+4}} .
\end{aligned}
$$

\section{APPENDIX C: EXTRACAVITY STROBOSCOPIC MEASUREMENT}

In this Appendix we provide more details about the derivation of expressions (19) and (20). Our starting point will be Eq. (17), where for simplicity we assume the ideal QND interaction given in Eq. (3). As described in Sec. V, we consider $\delta$-correlated quantum noise $\left[\hat{a}_{\mathrm{in}, t}, \hat{a}_{\mathrm{in}, t^{\prime}}^{\dagger}\right]=\delta(t-$ $\left.t^{\prime}\right)$. The operators $\hat{a}_{\text {in, } t}$ are singular and have dimension (time $)^{-1 / 2}$; Hermitian combinations of them thus cannot be directly associated with observables to be measured. To remedy that, it is customary to introduce the so-called quantum Wiener increment, defined as $\Delta \hat{W}_{\text {in }}=\int_{t}^{t+\tau} d t^{\prime} \hat{a}_{\text {in, } t^{\prime}}$. For a short interval of time we have $d \hat{W}_{\text {in }}=\hat{a}_{\text {in }, t} d t$ (obtained for $\tau \rightarrow d t$ ). From this expression one can define proper dimensionless modes $\hat{A}_{\text {in }}$ via $d \hat{W}_{\text {in }}=\hat{A}_{\text {in }} \sqrt{\tau}$, which are nonsingular $\left[\hat{A}_{\text {in }}, \hat{A}_{\text {in }}^{\dagger}\right]=1$ and can be associated with a measurement. The corresponding quadrature operators are $\hat{X}_{\text {in }}=$ $\left(\hat{A}_{\text {in }}+\hat{A}_{\text {in }}^{\dagger}\right) / \sqrt{2}, \hat{P}_{\text {in }}=i\left(\hat{A}_{\text {in }}^{\dagger}-\hat{A}_{\text {in }}\right) / \sqrt{2}$ and are associated with quadrature measurements of the outgoing light field. Since the expression in Eq. (18) still contains interactions which are bilinear, we can repeat the same analysis of Sec. II to get the conditional covariance matrix of the optomechanical system, now on the enlarged phase space with coordinate $\left(X_{\text {in }}, P_{\text {in }}, X_{c}, P_{c}, X_{m}, P_{m}\right)$.

\section{APPENDIX D: STROBOSCOPIC TOMOGRAPHY}

\section{General derivation}

We would like to model a series of BAE measurements on a damped harmonic oscillator in a thermal environment. To do so, we need to find the probability distribution of $\vec{x}$ given some measurement record $\vec{y}, P(\vec{x} \mid \vec{y})$, assuming that the parameters $\sigma_{m}, \sigma_{d}, \gamma$ are known.

From the description in the main text, we can determine the conditional probability distribution for the measurement outcomes,

$$
P(\vec{y} \mid \vec{x})=\prod_{i=0}^{N-1} \frac{1}{\sqrt{2 \pi \sigma_{m}^{2}}} \exp \left(-\frac{\left(y_{i}-x_{i}\right)^{2}}{2 \sigma_{m}^{2}}\right),
$$

as well as our prior

$$
P(\vec{x})=\frac{1}{\sqrt{2 \pi \sigma_{x_{0}}^{2}}} \exp \left(-\frac{x_{0}^{2}}{2 \sigma_{x_{0}}^{2}}\right)
$$

$$
\times \prod_{i=1}^{N-1} \frac{1}{\sqrt{2 \pi \sigma_{d}^{2}}} \exp \left(-\frac{\left(x_{i}-e^{-\gamma T / 2} x_{i-1}\right)^{2}}{2 \sigma_{d}^{2}}\right)
$$

The initial variance for $x_{0}$ could be from a thermal state. Optionally, we could already have performed measurements at that point, in which case the initial variance and mean are given by the resulting state. We can thus write down the joint probability distribution $P(\vec{x}, \vec{y})=P(\vec{y} \mid \vec{x}) P(\vec{x})$ :

$$
P(\vec{x}, \vec{y})=P(\vec{x}) P(\vec{y} \mid \vec{x}) \propto \exp \left(-\frac{1}{2}\left(\vec{x}^{\top}, \vec{y}^{\top}\right) \mathrm{Q}\left(\begin{array}{l}
\vec{x} \\
\vec{y}
\end{array}\right)\right),
$$

where $Q$ has entries $Q_{y y}=-Q_{x y}=\left(1 / \sigma_{m}^{2}\right) 1$, and

$$
\begin{aligned}
{\left[\mathrm{Q}_{x x}\right]_{i j}=} & -\frac{e^{-\gamma T / 2}}{\sigma_{d}^{2}}\left(\delta_{i, j+1}+\delta_{i, j-1}\right) \\
& +\left[\frac{1}{\sigma_{m}^{2}}+\frac{1+e^{-\gamma T}}{\sigma_{d}^{2}}-\delta_{i, N} \frac{e^{-\gamma T}}{\sigma_{d}^{2}}\right. \\
& \left.+\delta_{i, 1}\left(\frac{1}{\sigma_{x_{0}}^{2}}-\frac{1}{\sigma_{d}^{2}}\right)\right] \delta_{i, j} .
\end{aligned}
$$

$P(\vec{x}, \vec{y})$ is a normal distribution $(\vec{x}, \vec{y}) \sim N[\vec{\mu}, \Sigma]$, with mean $\vec{\mu}=0$ and covariance matrix $\Sigma=\mathrm{Q}^{-1}$, which can be found via block matrix inversion:

$$
\Sigma=\left(\begin{array}{cc}
\Sigma_{x x} & \Sigma_{x y} \\
\Sigma_{y x} & \Sigma_{y y}
\end{array}\right)
$$

where

$$
\begin{aligned}
& \Sigma_{y y}=\sigma_{m}^{2}+\Sigma_{x x}, \\
& \Sigma_{x x}=\left(Q_{x x}-1 / \sigma_{m}^{2}\right)^{-1}=\Sigma_{x y}=\Sigma_{y x} .
\end{aligned}
$$

Given this, the conditional distribution for $\vec{x}$ can be derived from the joint distribution

$$
\vec{x} \sim N\left(\Sigma_{x y} \Sigma_{y y}^{-1} \vec{y}, \Sigma_{x x}-\Sigma_{x y} \Sigma_{y y}^{-1} \Sigma_{x y}\right) .
$$

For now, we are only interested in the mean and variance of the first entry. We thus need to determine

$$
\Sigma_{x x} \Sigma_{y y}^{-1}=\left(\sigma_{m}^{2} \Sigma_{x x}^{-1}+1\right)^{-1}=\mathrm{Q}_{x x}^{-1} / \sigma_{m}^{2},
$$

and

$$
\Sigma_{x x}-\Sigma_{x y} \Sigma_{y y}^{-1} \Sigma_{x y}=\Sigma_{x x}\left(1-\sigma_{m}^{-2} \mathrm{Q}_{x x}^{-1}\right)=\mathrm{Q}_{x x}^{-1} .
$$

Note that $\Sigma_{x x}$ commutes with the matrix in rounded brackets. The best estimates for the positions $\vec{x}$ can therefore be 
obtained from the measurement results by multiplying the latter with the weights (D8) and their covariance is given through Eq. (D9). In both cases we need to determine $Q_{x x}^{-1}$, which is done in the following section.

\section{Explicit calculation of the inverse of the matrix $\mathbf{Q}_{x x}$}

To compute the inverse it is useful to consider the matrix

$$
\mathrm{M}=\left(\begin{array}{ccccccc}
1 & a & 0 & \cdots & 0 & 0 & 0 \\
a & b & a & \cdots & 0 & 0 & 0 \\
0 & a & b & \cdots & 0 & 0 & 0 \\
\vdots & \vdots & \vdots & \ddots & \vdots & \vdots & \vdots \\
0 & 0 & 0 & \cdots & b & a & 0 \\
0 & 0 & 0 & \cdots & a & b & a \\
0 & 0 & 0 & \cdots & 0 & a & 1
\end{array}\right) .
$$

We show in Appendix D 3 that, for experimentally relevant parameters, this coincides with a rescaled version of the matrix $\mathrm{Q}_{x x}$; namely, $\mathrm{M}=\mathrm{Q}_{x x} /\left[\mathrm{Q}_{x x}\right]_{11}$. The entries of the inverted matrix are [51]

$$
M_{i j}^{-1}=(-1)^{i+j} a^{|i-j|} \begin{cases}\theta_{i-1} \phi_{j+1} / \theta_{n} & \text { if } i \leqslant j \\ \theta_{j-1} \phi_{i+1} / \theta_{n} & \text { if } i \geqslant j,\end{cases}
$$

where $\theta$ and $\phi$ fulfill certain recurrence relations. In our case, they are in fact the same, and we have

$$
\begin{array}{ll}
\theta_{i}=b \theta_{i-1}-a^{2} \theta_{i-2}, & \theta_{0}=\theta_{1}=1, \\
\theta_{n}=\theta_{n-1}-a^{2} \theta_{n-2}, & \phi_{i}=\theta_{n+1-i} .
\end{array}
$$

The solution can be found from the characteristic polynomial $x^{2}-b x+a^{2}=0$, which has the roots

$$
\xi_{ \pm}=\frac{b}{2} \pm \sqrt{\frac{b^{2}}{4}-a^{2}} .
$$

Fitting the general solution to the boundary conditions, we find

$$
\theta_{i}=\frac{1}{\sqrt{b^{2}-4 a^{2}}}\left[\xi_{-}^{i}\left(\xi_{+}-1\right)-\xi_{+}^{i}\left(\xi_{-}-1\right)\right],
$$

but with $\theta_{n}$ determined by Eq. (D12) above. This allows us to write the inverse of the above matrix in an exact analytical, albeit unwieldy, manner

$$
\begin{aligned}
\left(\mathrm{M}^{-1}\right)_{i j} & =\frac{(-1)^{i+j} a^{|i-j|}}{\sqrt{b^{2}-4 a^{2}}} \frac{v(i-1) v(n-j)}{v(n-1)-a^{2} v(n-2)}, \\
v(i) & \equiv \xi_{-}^{i}\left(\xi_{+}-1\right)-\xi_{+}^{i}\left(\xi_{-}-1\right) .
\end{aligned}
$$

This exact formula for the inverse represents the central result of this Appendix. We consider simplifications that arise in certain cases below.

\section{a. Physically relevant case}

The relevant case is $b^{2} / 4>a^{2}$. In this case $\xi_{+}>\xi_{-}$, such that, in the limit of a large number of measurements $n \rightarrow \infty$, the formula for the inverse turns into

$$
\begin{aligned}
\left(\mathrm{M}^{-1}\right)_{i j} & =\frac{(-1)^{i+j} a^{|i-j|}}{\sqrt{b^{2}-4 a^{2}}} \frac{v(i-1)}{\xi_{+}^{j-1}-a^{2} \xi_{+}^{j-2}}, \quad i<j \\
\mathrm{M}_{j i} & =\mathrm{M}_{i j}, \quad i>j .
\end{aligned}
$$

It is only exact for $n \rightarrow \infty$ and is a good approximation if $\left(a \xi_{-} / \xi_{+}\right)^{n}$ is small. The weights for the measurement of the initial state are the special case $i=1$, i.e.,

$$
\left(\mathrm{M}^{-1}\right)_{1 j} \simeq \frac{(-a)^{|1-j|}}{\xi_{+}^{j-1}-a^{2} \xi_{+}^{j-2}} .
$$

Another useful special case is $i=j$, in which case Eq. (D16) simplifies to

$$
\left(\mathrm{M}^{-1}\right)_{i i}=\frac{\left(\xi_{-} / \xi_{+}\right)^{i-1}\left(\xi_{+}-1\right)-\xi_{-}+1}{\sqrt{b^{2}-4 a^{2}}\left(1-a^{2} / \xi_{+}\right)} .
$$

On the other hand, the variance in the steady state $(i \rightarrow \infty$ but $n / i \gg 1$ ), which corresponds to the variance when taking all measurements before and after a specific point in time into account,

$$
\lim _{n \rightarrow \infty}\left(\mathrm{M}^{-1}\right)_{n / 2, n / 2}=\frac{1-\xi_{-}}{\sqrt{b^{2}-4 a^{2}}\left(1-a^{2} / \xi_{+}\right)} .
$$

\section{b. Other cases}

For completeness, we mention the other case $b^{2} / 4<a^{2}$, which implies $\xi_{-}=\xi_{+}^{*} \equiv \xi$, such that

$$
\left(\mathrm{M}^{-1}\right)_{i j}=\frac{(-1)^{i+j} a^{|i-j|}}{\sqrt{b^{2}-4 a^{2}}} \frac{2 \operatorname{Im}\left[\xi^{i-1}\left(\xi^{*}-1\right)\right] \operatorname{Im}\left[\xi^{n-j}\left(\xi^{*}-1\right)\right]}{i \operatorname{Im}\left[\xi^{n-2}\left(\xi^{*}-1\right)-a^{2} \xi^{n-1}\left(\xi^{*}-1\right)\right]},
$$

where $\operatorname{Im}[x]$ denotes the imaginary part of $x$.

Finally, if $b^{2} / 4=a^{2}$, the matrix is not invertible.

\section{Variance and measurement weights for experimentally relevant parameters}

The actual matrix we are interested in has parameters

$$
\begin{aligned}
{\left[\mathrm{Q}_{x x}\right]_{11} } & =\frac{1}{\sigma_{x_{0}}^{2}}+\frac{1}{\sigma_{m}^{2}}+\frac{e^{-\gamma T}}{\sigma_{d}^{2}}, \\
{\left[\mathrm{Q}_{x x}\right]_{i, i+1} } & =-\frac{e^{-\gamma T / 2}}{\sigma_{d}^{2}}, \\
{\left[\mathrm{Q}_{x x}\right]_{i i} } & =\frac{1}{\sigma_{m}^{2}}+\frac{1+e^{-\gamma T}}{\sigma_{d}^{2}}, \\
{\left[\mathrm{Q}_{x x}\right]_{n n} } & =\frac{1}{\sigma_{m}^{2}}+\frac{1}{\sigma_{d}^{2}} .
\end{aligned}
$$

To use the analytical matrix inverse derived in Appendix D2, we define $\mathrm{M}=\mathrm{Q}_{x x} /\left[\mathrm{Q}_{x x}\right]_{11}$, which has $\mathrm{M}_{11}=1, \quad \mathrm{M}_{n n} \approx 1, \quad \mathrm{M}_{i, i+1}=a=\left[\mathrm{Q}_{x x}\right]_{i, i+1} /\left[\mathrm{Q}_{x x}\right]_{11}$, and $\mathrm{M}_{i i}=b=\left[\mathrm{Q}_{x x}\right]_{i i} /\left[\mathrm{Q}_{x x}\right]_{11}$. The fact that the last element of the diagonal of $\mathrm{M}$ is not 1 is irrelevant if the number $n$ of measurements is large. We can therefore take it to be 1 for simplicity. Technically, the matrix is still invertible without this assumption, but it leads to cumbersome formulas that are not very enlightening.

For stroboscopic measurements to make sense, we require $\sigma_{d}^{2} \ll 1$, i.e., the state is coherent for several periods. As a result, the term with $1 / \sigma_{d}^{2}$ dominates all the elements of $\mathrm{Q}_{x x}$. Physically, this means that the value of $x_{i}$ is most strongly constrained by its neighbors $x_{i-1}$ and $x_{i+1}$, and much less by the measurement or our initial guess. This is precisely the 
regime of a slowly decohering and weakly measured oscillator that we consider here. In this limit,

$$
b^{2} / 4-a^{2}=\mathrm{Q}_{x x, 11}^{-2}\left[\frac{1}{4 \sigma_{m}^{2}}+\frac{1+e^{-\gamma T}}{2 \sigma_{m}^{2} \sigma_{d}^{2}}-\frac{e^{-\gamma T}}{\sigma_{d}^{4}}\right]>0,
$$

such that we may use the formulas from Appendix D2a.

To leading order in $\sigma_{d}, \mathrm{M}$ is the discrete Laplace operator, with $a=1$ and $b=2$, which is not invertible, so we have to go to next order to get physical answers. Note that $\gamma T=$ $\sigma_{d}^{2} /\left(n_{\text {th }}+1 / 2\right)$, such that

$$
\begin{aligned}
& a=\frac{\left[\mathrm{Q}_{x x}\right]_{i, i+1}}{\left[\mathrm{Q}_{x x}\right]_{11}} \simeq-1+\sigma_{d}^{2}\left(\frac{1}{\sigma_{m}^{2}}+\frac{1}{\sigma_{x_{0}}^{2}}-\frac{1}{2 n_{\mathrm{th}}+1}\right), \\
& b=\frac{\left[\mathrm{Q}_{x x}\right]_{i i}}{\left[\mathrm{Q}_{x x}\right]_{11}} \simeq 2+\sigma_{d}^{2}\left(\frac{2}{2 n_{\mathrm{th}}+1}-\frac{1}{\sigma_{m}^{2}}-\frac{2}{\sigma_{x_{0}}^{2}}\right) .
\end{aligned}
$$

Using the formulas for the inverse of the matrix derived above, we can now calculate the variance of our measurement of the initial state $(i=1)$ :

$$
Q_{x x, 11}^{-1}\left(\mathrm{M}^{-1}\right)_{11}=\sqrt{\frac{\pi\left(1 / 2+n_{\mathrm{th}}\right)}{2 Q \chi^{2}}}+O\left(Q^{-3 / 2}\right),
$$

where we have used

$$
\sigma_{m}^{2}=1 /\left(2 \chi^{2}\right)
$$

for the measurement we consider. This expression coincides with the amount of squeezing predicted in Eq. (13).

On the other hand, the variance in steady-state $(i \rightarrow \infty$ but $n / i \gg 1$ ), Eq. (D19), simplifies to

$$
\lim _{n \rightarrow \infty}\left(\mathrm{M}^{-1}\right)_{n / 2, n / 2}=\sqrt{\frac{\pi\left(1 / 2+n_{\mathrm{th}}\right)}{8 Q \chi^{2}}}+O\left(Q^{-3 / 2}\right),
$$

i.e., to leading order it is just half of Eq. (D24).
[1] M. Aspelmeyer, T. J. Kippenberg, and F. Marquardt, Rev. Mod. Phys. 86, 1391 (2014).

[2] J. Chan, T. P. M. Alegre, A. H. Safavi-Naeini, J. T. Hill, A. Krause, S. Gröblacher, M. Aspelmeyer, and O. Painter, Nature (London) 478, 89 (2011).

[3] J. D. Teufel, T. Donner, D. Li, J. W. Harlow, M. S. Allman, K. Cicak, A. J. Sirois, J. D. Whittaker, K. W. Lehnert, and R. W. Simmonds, Nature (London) 475, 359 (2011).

[4] E. E. Wollman, C. U. Lei, A. J. Weinstein, J. Suh, A. Kronwald, F. Marquardt, A. A. Clerk, and K. C. Schwab, Science 349, 952 (2015).

[5] F. Lecocq, J. B. Clark, R. W. Simmonds, J. Aumentado, and J. D. Teufel, Phys. Rev. X 5, 041037 (2015).

[6] J.-M. Pirkkalainen, E. Damskägg, M. Brandt, F. Massel, and M. A. Sillanpää, Phys. Rev. Lett. 115, 243601 (2015).

[7] C. F. Ockeloen-Korppi, E. Damskägg, J. M. Pirkkalainen, M. Asjad, A. A. Clerk, F. Massel, M. J. Woolley, and M. A. Sillanpää, Nature (London) 556, 478 (2018).

[8] S. G. Hofer, W. Wieczorek, M. Aspelmeyer, and K. Hammerer, Phys. Rev. A 84, 052327 (2011).

[9] S. Machnes, J. Cerrillo, M. Aspelmeyer, W. Wieczorek, M. B. Plenio, and A. Retzker, Phys. Rev. Lett. 108, 153601 (2012).

[10] J.-Q. Liao and C. K. Law, Phys. Rev. A 84, 053838 (2011).

[11] N. Vostrosablin, A. A. Rakhubovsky, and R. Filip, Phys. Rev. A 94, 063801 (2016).

[12] T. A. Palomaki, J. D. Teufel, R. W. Simmonds, and K. W. Lehnert, Science 342, 710 (2013).

[13] T. A. Palomaki, J. W. Harlow, J. D. Teufel, R. W. Simmonds, and K. W. Lehnert, Nature (London) 495, 210 (2013).

[14] M. R. Vanner, I. Pikovski, G. D. Cole, M. S. Kim, Č. Brukner, K. Hammerer, G. J. Milburn, and M. Aspelmeyer, Proc. Natl. Acad. Sci. USA 108, 16182 (2011).

[15] J. S. Bennett, K. Khosla, L. S. Madsen, M. R. Vanner, H. Rubinsztein-Dunlop, and W. P. Bowen, New J. Phys. 18, 053030 (2016).

[16] U. B. Hoff, J. Kollath-Bönig, J. S. Neergaard-Nielsen, and U. L. Andersen, Phys. Rev. Lett. 117, 143601 (2016).
[17] J. S. Bennett and W. P. Bowen, New J. Phys. 20, 113016 (2018).

[18] N. Vostrosablin, A. A. Rakhubovsky, U. B. Hoff, U. L. Andersen, and R. Filip, New J. Phys. 20, 083042 (2018).

[19] K. E. Khosla, G. A. Brawley, M. R. Vanner, and W. P. Bowen, Optica 4, 1382 (2017).

[20] M. Asjad, G. S. Agarwal, M. S. Kim, P. Tombesi, G. Di Giuseppe, and D. Vitali, Phys. Rev. A 89, 023849 (2014).

[21] J. Clarke, P. Sahium, K. E. Khosla, I. Pikovski, M. S. Kim, and M. R. Vanner, arXiv:1910.09603.

[22] M. R. Vanner, J. Hofer, G. D. Cole, and M. Aspelmeyer, Nat. Commun. 4, 2295 (2013).

[23] J. T. Muhonen, G. R. La Gala, R. Leijssen, and E. Verhagen, Phys. Rev. Lett. 123, 113601 (2019).

[24] D. Malz and A. Nunnenkamp, Phys. Rev. A 94, 023803 (2016).

[25] L. Qiu, I. Shomroni, M. A. Ioannou, N. Piro, D. Malz, A. Nunnenkamp, and T. J. Kippenberg, Phys. Rev. A 100, 053852 (2019).

[26] V. B. Braginsky, Y. I. Vorontsov, and F. Y. Khalili, JETP Lett. 27, 296 (1978).

[27] L. E. Marchese, M. F. Bocko, and R. Onofrio, Phys. Rev. D 45, 1869 (1992).

[28] R. Onofrio, Phys. Lett. A 148, 1 (1990).

[29] G. Vasilakis, V. Shah, and M. V. Romalis, Phys. Rev. Lett. 106, 143601 (2011).

[30] G. Vasilakis, H. Shen, K. Jensen, M. Balabas, D. Salart, B. Chen, and E. S. Polzik, Nat. Phys. 11, 389 (2015).

[31] C. M. Caves, K. S. Thorne, R. W. P. Drever, V. D. Sandberg, and M. Zimmermann, Rev. Mod. Phys. 52, 341 (1980).

[32] M. F. Bocko and R. Onofrio, Rev. Mod. Phys. 68, 755 (1996).

[33] C. Weedbrook, S. Pirandola, R. García-Patrón, N. J. Cerf, T. C. Ralph, J. H. Shapiro, and S. Lloyd, Rev. Mod. Phys. 84, 621 (2012).

[34] C. M. Caves and G. J. Milburn, Phys. Rev. A 36, 5543 (1987).

[35] S. Olivares, Eur. Phys. J. Spec. Top. 203, 3 (2012).

[36] M. G. Genoni, L. Lami, and A. Serafini, Contemp. Phys. 57, 331 (2016).

[37] A. Serafini, Quantum Continuous Variables: A Primer of Theoretical Methods (CRC Press, Boca Raton, 2017). 
[38] G. Giedke and J. Ignacio Cirac, Phys. Rev. A 66, 032316 (2002).

[39] M. Brunelli, D. Malz, and A. Nunnenkamp, Phys. Rev. Lett. 123, 093602 (2019).

[40] S. Gammelmark, B. Julsgaard, and K. Mølmer, Phys. Rev. Lett. 111, 160401 (2013)

[41] J. Zhang and K. Mølmer, Phys. Rev. A 96, 062131 (2017).

[42] M. Rossi, D. Mason, J. Chen, and A. Schliesser, Phys. Rev. Lett. 123, 163601 (2019).

[43] K. Hammerer, M. Aspelmeyer, E. S. Polzik, and P. Zoller, Phys. Rev. Lett. 102, 020501 (2009).

[44] M. J. Woolley and A. A. Clerk, Phys. Rev. A 87, 063846 (2013).
[45] E. S. Polzik and K. Hammerer, Ann. Phys. (Berlin, Ger.) 527, A15 (2015).

[46] M. Rossi, D. Mason, J. Chen, Y. Tsaturyan, and A. Schliesser, Nature (London) 563, 53 (2018).

[47] Y. Tsaturyan, A. Barg, E. S. Polzik, and A. Schliesser, Nat. Nanotechnol. 12, 776 (2017).

[48] C. Reinhardt, T. Müller, A. Bourassa, and J. C. Sankey, Phys. Rev. X 6, 021001 (2016).

[49] R. A. Norte, J. P. Moura, and S. Gröblacher, Phys. Rev. Lett. 116, 147202 (2016).

[50] L. Qiu, I. Shomroni, P. Seidler, and T. Kippenberg, Phys. Rev. Lett. 124, 173601 (2020).

[51] R. A. Usmani, Lin. Alg. Appl. 212-213, 413 (1994). 